\title{
Mechanism Prediction of Astragalus membranaceus against Cisplatin-Induced Kidney Damage by Network Pharmacology and Molecular Docking
}

\author{
Congchao Jia $\mathbb{D}^{1},{ }^{1}$ Xianchao Pan $\mathbb{D},{ }^{2}$ Binyou Wang $\mathbb{D},{ }^{3}$ Pengyu Wang $\mathbb{D},{ }^{1}$ Yiwei Wang $\mathbb{D},{ }^{4,5}$ \\ and Rong Chen ${ }^{6}$ \\ ${ }^{1}$ Clinical Medical College, Southwest Medical University, Luzhou, Sichuan 646000, China \\ ${ }^{2}$ Department of Medicinal Chemistry, College of Pharmacy, Southwest Medical University, Luzhou, Sichuan 646000, China \\ ${ }^{3}$ College of Pharmacy, Southwest Medical University, Luzhou, Sichuan 646000, China \\ ${ }^{4}$ State Key Laboratory of Biotherapy, West China Hospital, Sichuan University, Chengdu 610041, China \\ ${ }^{5}$ College of Basic Medical Sciences, Southwest Medical University, Luzhou 646000, China \\ ${ }^{6}$ Department of Pathophysiology, College of Basic Medical Sciences, Southwest Medical University, Luzhou 646000, China
}

Correspondence should be addressed to Rong Chen; cr0204@swmu.edu.cn

Received 27 April 2021; Revised 21 July 2021; Accepted 11 August 2021; Published 19 August 2021

Academic Editor: Yanggang Yuan

Copyright (C) 2021 Congchao Jia et al. This is an open access article distributed under the Creative Commons Attribution License, which permits unrestricted use, distribution, and reproduction in any medium, provided the original work is properly cited.

\begin{abstract}
Background. Cisplatin is a frequently used and effective chemotherapy drug in clinical practice, but severe side effects limit its use, among which nephrotoxicity is considered the most serious and prolonged damage to the body. Astragalus membranaceus (AM) is a well-known herbal medicine, and modern pharmacological studies have confirmed its antioxidant, immunomodulatory, and antiapoptotic effects. Clinical studies have shown that AM and its active components can attenuate cisplatin-induced kidney damage, but the molecular mechanism has not been fully expounded. Materials and Methods. First, the components and targets information of AM were collected from the TCMSP, and the relevant targets of cisplatin-induced kidney damage were accessed from the GeneCards and OMIM databases. Then, the core targets were selected by the Venn diagram and network topology analysis, which was followed by GO and KEGG pathway enrichment analysis. Finally, we construct a component-target-pathway network. Furthermore, molecular docking was carried out to identify the binding activity between active components and key targets. Results. A total of 20 active components and 200 targets of AM and 646 targets related to cisplatin-induced kidney damage were obtained. 91 intersection targets were found between AM and cisplatin-induced kidney damage. Then, 16 core targets were identified, such as MAPK1, TNF- $\alpha$, and p53. Furthermore, GO and KEGG pathway enrichment analysis suggested that MAPK, Toll-like receptor, and PI3K-Akt signaling pathways may be of significance in the treatment of cisplatin-induced kidney damage by AM. Molecular docking indicated that quercetin and kaempferol had high binding affinities with many core targets. Conclusion. In summary, the active components, key targets, and signaling pathways of AM in the treatment of cisplatin-induced kidney damage were predicted in this study, which contributed to the development and application of AM.
\end{abstract}

\section{Introduction}

Cisplatin, a heavy metallic compound with powerful anticancer effects, is synergistic with many antitumor drugs without cross-resistance, thus being one of the most frequently used and effective chemotherapy drugs at present [1]. However, it has many serious side effects that greatly limit its therapeutic use, including gastric toxicity, ototoxicity, nephrotoxicity, allergic reactions, and bone marrow suppression [2]. Nephrotoxicity of cisplatin is considered the most serious and prolonged damage to the body [3]. Acute kidney injury (AKI) has been reported in $25-30 \%$ of patients roughly, who have received cisplatin chemotherapy [4]. The mechanisms of cisplatin-induced kidney damage are very complicated, involving multiple factors such as oxidative damage, dysfunctional 
mitochondria, and renal tubular epithelial cell apoptosis [5-9]. Although hydration diuresis and diuretic forced diuresis are commonly used clinically to reduce cisplatininduced kidney damage, the results are still unsatisfactory $[10,11]$. Therefore, there is an immediate need to pursue an effective measure to mitigate cisplatin-induced kidney damage.

Astragalus membranaceus (AM), also known as "Huang Qi" in traditional Chinese medicine, is the dried root of membranous Astragalus or Astragalus mongolicus, which has been widely used in the clinical setting [12]. AM, which has a long history in China, was first recorded in Shennong's Herbal Classic (an ancient classic Chinese herbal medicine book). Modern pharmacological studies have shown that AM contains flavonoids, saponins, and other active ingredients, which have a wide range of pharmacological effects, such as antioxidant, immune regulation, antiapoptosis, and antitumor [13-16]. A recent study indicated that Astragalus polysaccharide (an active ingredient of AM) could reduce AKI induced by cisplatin through protecting mitochondria, inhibiting oxidative damage, and improving mitochondriamediated apoptosis [17]. Also, according to a meta-analysis, AM injection can alleviate the clinical side effects of platinum-based chemotherapy drugs [18]. However, the targets and specific mechanism of AM against cisplatin-induced kidney damage remain largely unknown.

Recently, network pharmacology has been a potent tool for gaining insights into the mechanisms of drugs [19]. This method explores the drug-component-target-pathway-disease relationship from a systems biology perspective, predicts potential drug treatment mechanisms for diseases, and provides a theoretical basis for the mechanisms of Chinese medicine treatment for diseases [20]. Molecular docking is an efficient computational method for the prediction of the binding affinity between a ligand and a target protein, which can be used to identify potential drug targets [21]. In this paper, the possible targets and the molecular mechanism of AM on the treatment of cisplatin-induced kidney damage were investigated by network pharmacology and molecular docking (Figure 1).

\section{Materials and Methods}

2.1. Screening Main Active Components and Targets of AM. Information of the active components and targets associated with AM was collected from the TCMSP database (https:// tcmspw.com/, February 20, 2021) [22]. The TCMSP database is a systematized pharmacology resource that provides the ADME (absorption, distribution, metabolism, and excretion) characteristics of Chinese herbal medicines or ingredients including oral bioavailability (OB), drug likeness (DL), blood-brain barrier, Caco-2 permeability, etc. [23]. Among the pharmacokinetic properties mentioned above, $\mathrm{OB}$ and DL are the most vital ones for drug delivery, as they help assess the effect of drug distribution in the circulatory system and how drug-like compounds regard factors such as bioavailability [24]. In the TCMSP database, OB is based on the internal model OBioavail 1.1 and DL is evaluated according to molecular descriptors and Tanimoto coefficients $[22,25]$. In this study, the name of the herb "Huang Qi" was inputted into the searching box to get the active components and related targets. We selected active components with $\mathrm{OB} \geq 30 \%$ and $\mathrm{DL} \geq 0.18$ and their corresponding targets. All targets associated with these active ingredients (both validated and predicted targets) were then imported into the UniProt database (https://www.uniprot. org/, February 15, 2021) to get the gene symbol of targets.

2.2. Targets Collection of Cisplatin-Induced Kidney Damage. By using the keywords of "cisplatin-induced kidney damage," "cisplatin-induced nephrotoxicity," "cisplatin kidney damage," and "cisplatin-induced kidney injury," gene symbols of disease-related targets were obtained from the OMIM (https://omim.org) and the GeneCards (https:// www.genecards.org) databases [26-28]. The results accessed from GeneCards were screened for the relevance score $\geq 10.00$ as the screening index. We chose Gene Map in "Advanced Search" to search target genes related to disease in the OMIM database. The ultimate disease-related targets were obtained by removing the duplicates.

\subsection{Screening Core Targets of AM in Cisplatin-Induced Kidney} Damage Treatment. The VENNY 2.1 online platform (https://bioinfogp.cnb.csic.es/tools/venny/) was used for intersection targets between $\mathrm{AM}$ and cisplatin-induced kidney damage. The intersection targets were used to constitute the protein-protein interaction (PPI) network using the STRING database (https://string-db.org/, February 28, 2021) [29] with confidence scores larger than 0.7 , and the other variables were left at their original values. We then exported the PPI network in TSV format and analyzed its topology properties (degree centrality) using Cytoscape 3.8.0 (https://cytoscape.org/) [30]. Degree centrality (DC) is the most commonly used topological parameter, which is utilized to appraise the central attribute of nodes in the network. The targets with the DC $\geq 2 \times$ median were selected as the core targets.

2.4. Constructing and Analyzing PPI Network of Core Targets. The core targets obtained above were inputted into the STRING database to get the PPI network. All settings were the same as above. Then, the PPI network was extracted in TSV format and inputted into Cytoscape 3.8.0 for further analysis.

2.5. Functional and Pathway Enrichment Analysis of Core Targets. To investigate the multiple mechanisms of AM against cisplatin-induced kidney damage at the systematic level further, we used DAVID 6.8 (https://david.ncifcrf.gov/, March 5, 2021) [31] to perform the functional enrichment analysis of core targets, including the Gene Ontology (GO) terms as well as Kyoto Encyclopedia of Genes and Genomes (KEGG) pathways [32, 33]. 


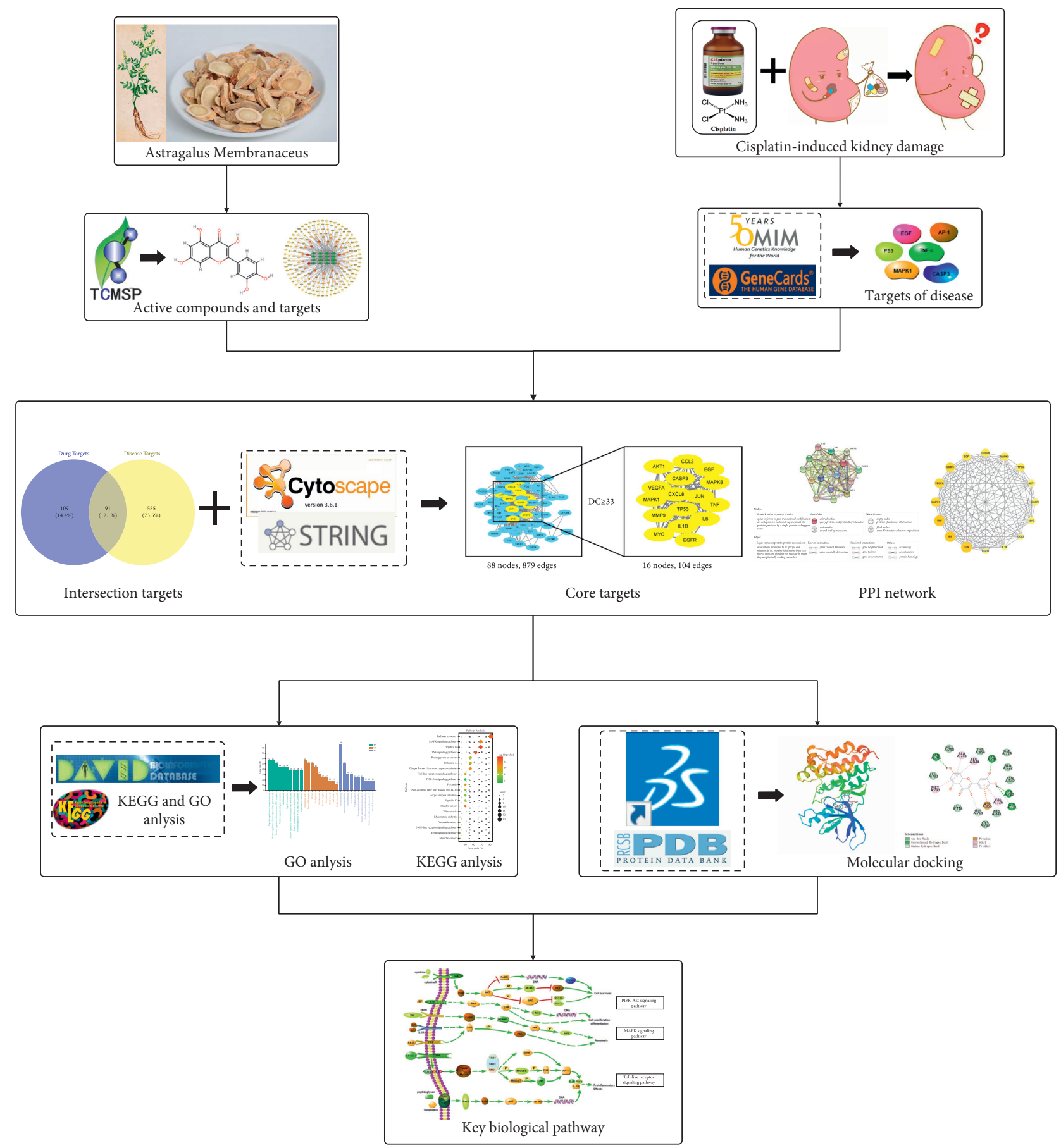

Figure 1: The workflow of this study.

2.6. AM Active Component-Target-Pathway Analysis. To investigate the relationship between active components, targets, and pathways, we constructed the network of active component-target-pathway (C-T-P network). We imported active components, targets, and pathways into Cytoscape 3.8.0 as three types of nodes and connected associated nodes with edges to construct the active components-targetspathways network.
2.7. Molecular Docking. We used molecular docking to predict the binding activity between the active ingredient and the core target. The structural formula of active components was obtained from PubChem (https://pubchem. ncbi.nlm.nih.gov/, March 10, 2021) and stored in SDF format. Afterward, the core target conformations were obtained from the Protein Data Bank database (PDB, https:// www.rcsb.org/, March 10, 2021). The following were the 
TABle 1: Database and software summary.

\begin{tabular}{lc}
\hline Names of database and software & Website \\
\hline Traditional Chinese Medicine Systems Pharmacology (TCMSP) database & https://tcmspw.com/ \\
UniPort database & https://www.uniprot.org/ \\
Online Mendelian Inheritance in Man (OMIM) & https:/omim.org/ \\
GeneCards database & https://www.genecards.org/ \\
VENNY 2.1 online platform & https://bioinfogp.cnb.csic.es/tools/venny/ \\
Cytoscape 3.8.0 & https://cytoscape.org/ \\
STRING database & https://string-db.org/ \\
DAVID 6.8 & https://david.ncifcrf.gov/ \\
PubChem & https://pubchem.ncbi.nlm.nih.gov/ \\
RCSB Protein Data Bank & https://www.rcsb.org/ \\
\hline
\end{tabular}

TABLE 2: Information about active components of AM.

\begin{tabular}{|c|c|c|c|c|}
\hline Mol ID & Molecule name & OB (\%) & $\mathrm{DL}$ & $\mathrm{DC}$ \\
\hline MOL000098 & Quercetin & 46.43 & 0.28 & 144 \\
\hline MOL000422 & Kaempferol & 41.88 & 0.24 & 58 \\
\hline MOL000378 & 7-O-Methylisomucronulatol & 74.69 & 0.30 & 43 \\
\hline MOL000392 & Formononetin & 69.67 & 0.21 & 36 \\
\hline MOL000354 & Isorhamnetin & 49.60 & 0.31 & 33 \\
\hline MOL000371 & 3,9-Di-O-methylnissolin & 53.74 & 0.48 & 22 \\
\hline MOL000296 & Hederagenin & 36.91 & 0.75 & 20 \\
\hline MOL000380 & $(6 \mathrm{aR}, 11 \mathrm{aR})-9,10-$ Dimethoxy-6a,11a-dihydro-6H-benzofurano [3,2-c]chromen-3-ol & 64.26 & 0.42 & 20 \\
\hline MOL000417 & Calycosin & 47.75 & 0.24 & 20 \\
\hline MOL000239 & Jaranol & 50.83 & 0.29 & 11 \\
\hline MOL000387 & Bifendate & 31.10 & 0.67 & 5 \\
\hline MOL000433 & FA & 68.96 & 0.71 & 3 \\
\hline MOL000442 & 1,7-Dihydroxy-3,9-dimethoxy pterocarpene & 39.05 & 0.48 & 3 \\
\hline MOL000379 & 9,10-Dimethoxypterocarpan-3-O- $\beta$-D-glucoside & 36.74 & 0.92 & 2 \\
\hline MOL000033 & $\begin{array}{l}\text { (3S,8S,9S,10R,13R,14S,17R)-10,13-Dimethyl-17-[(2R,5S)-5-propan-2-yloctan-2-yl]- } \\
2,3,4,7,8,9,11,12,14,15,16,17 \text {-dodecahydro-1H-cyclopenta[a]phenanthren-3-ol }\end{array}$ & 36.23 & 0.78 & 1 \\
\hline MOL000211 & Mairin & 55.38 & 0.78 & 1 \\
\hline MOL000374 & $5^{\prime}$-Hydroxyiso-muronulatol-2' $5^{\prime}$-di-O-glucoside & 41.72 & 0.69 & 0 \\
\hline MOL000398 & Isoflavanone & 109.99 & 0.3 & 0 \\
\hline MOL000438 & (3R)-3-(2-Hydroxy-3,4-dimethoxyphenyl)chroman-7-ol & 67.67 & 0.26 & 0 \\
\hline MOL000439 & Isomucronulatol- $7,2^{\prime}$-di-O-glucosiole & 49.28 & 0.62 & 0 \\
\hline
\end{tabular}

screening conditions: (1) The biological source of protein structure is human; (2) The protein structure is acquired by $\mathrm{X}$-crystal diffraction; (3) The resolution of protein crystals is less than $3 \AA$; and (4) Protein structures with unique ligands are preferred. The structures of the ligands and the protein receptors were prepared by using Discovery Studio (DS, BIOVIA, 2019). Then, we used the flexible docking procedure CDOCKER built-in DS for molecular docking with CHARMM force field (Chemistry at HARvard Macromolecular Mechanics).

2.8. Database and Software Summary. The databases and software used in this study are listed in Table 1 for the convenience of readers.

\section{Results}

3.1. Active Components and Targets of AM. We gained 86 components from the TCMSP database, mainly including quercetin, isoflavanone, kaempferol, and so forth. A total of
20 active components satisfied the criteria of $\mathrm{OB} \geq 30 \%$ and $\mathrm{DL} \geq 0.18$ (Table 2). Then, we obtained a total of 200 targets related to these 20 active components from TCMSP. Figure 2 shows the interaction between active components and targets. It could be seen that quercetin had the maximal DC, that is to say, quercetin had the most targets, suggesting that quercetin might be an important active component of AM.

3.2. Targets Related to Cisplatin-Induced Kidney Damage. We found 534 relevant targets of cisplatin-induced kidney damage in GeneCards and 121 targets in OMIM databases. After deleting duplicate targets, 646 disease targets were finally obtained. The top 5 targets for relevance score were cellular tumor antigen p53 (p53), tumor necrosis factoralpha (TNF- $\alpha$ ), interleukin-6 (IL-6), angiotensin-converting enzyme, and transforming growth factor-beta 1 .

3.3. Core Targets of AM in Cisplatin-Induced Kidney Damage Treatment. The Venn diagram showed that there were 91 intersection targets of AM and cisplatin-induced kidney 


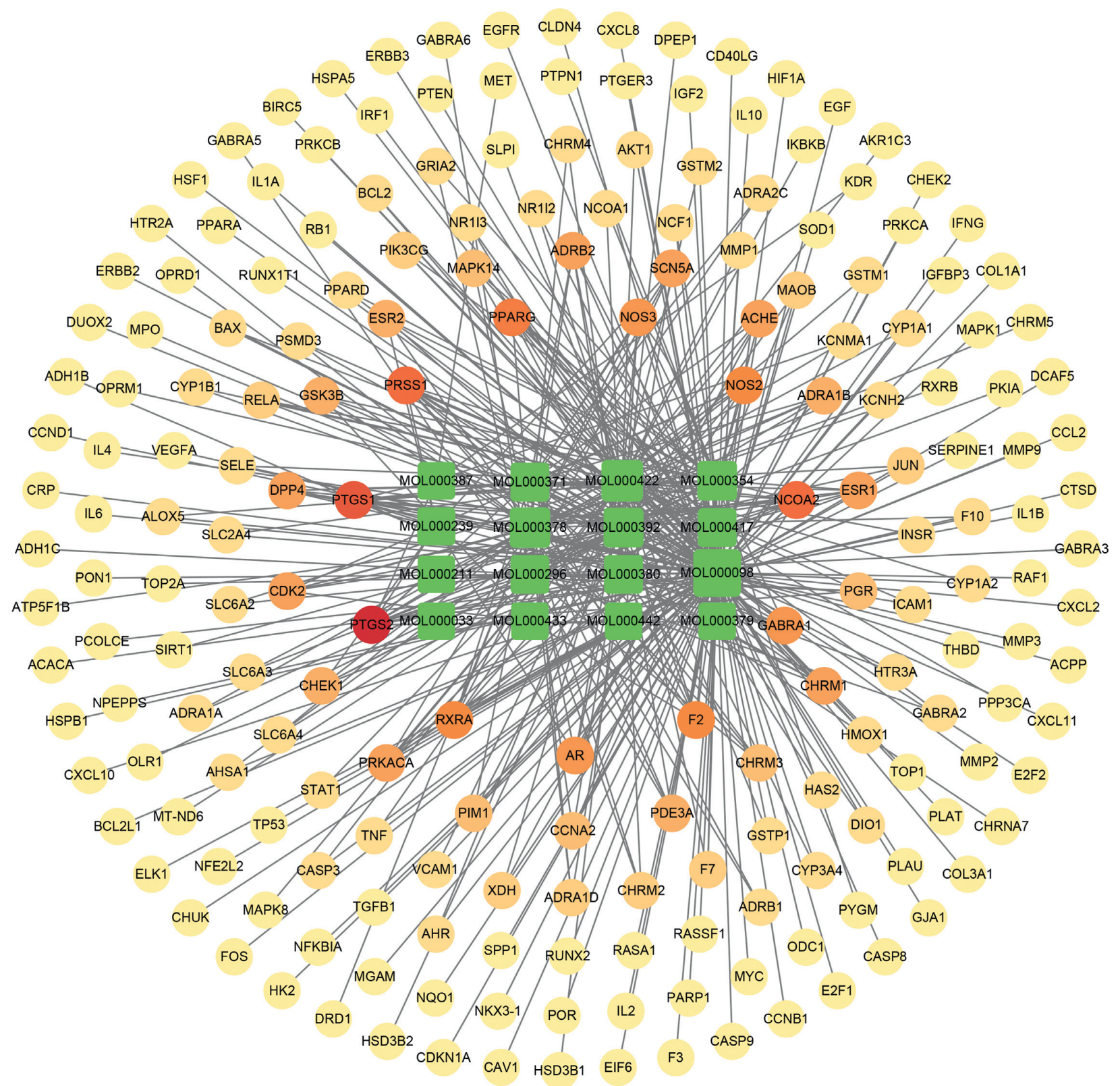

FIGURE 2: Network of active component-target. The green square means the active components of AM, and its DC value is represented by the node size. The circular node means the active components' targets, and a darker node color indicates a larger degree value. The interaction between the components and the targets is represented by the edge.

damage (Figure 3). The intersection targets were used to construct the PPI network using the STRING data set. Immediately afterward, the network was introduced into Cytoscape 3.8.0 and the DC of each node was calculated by using the function of "network analyzer." 16 core targets of $\mathrm{AM}$ in cisplatin-induced kidney damage treatment were singled out with DC $\geq 33(2 \times 16.5)$ (Figure 4$)$. Table 3 shows the specific information of the 16 core targets.

3.4. Constructing and Analyzing PPI Network. The 16 core targets were introduced into the STRING database to get the PPI network (Figure 5(a)). The DC was reckoned with "Network Analyzer" in Cytoscape 3.8.0 to reflect the significance of a target in the network. Significant targets are indicated by darker colors, larger sizes, and higher DC of interaction in the network, such as mitogen-activated protein kinase 1 (MAPK1), TNF- $\alpha$, IL-6, vascular endothelial growth factor A (VEGFA) (Figure 5(b)).

3.5. GO and KEGG Enrichment Analysis. For the purpose of figuring out the mechanism of AM against cisplatin-induced kidney damage more systematically, the 16 core targets were inputted into DAVID 6.8 to GO and KEGG enrichment analysis. There were 186 items related to biological processes, mainly including MAPK cascade, positive regulation of smooth muscle cell proliferation, inflammatory response, 


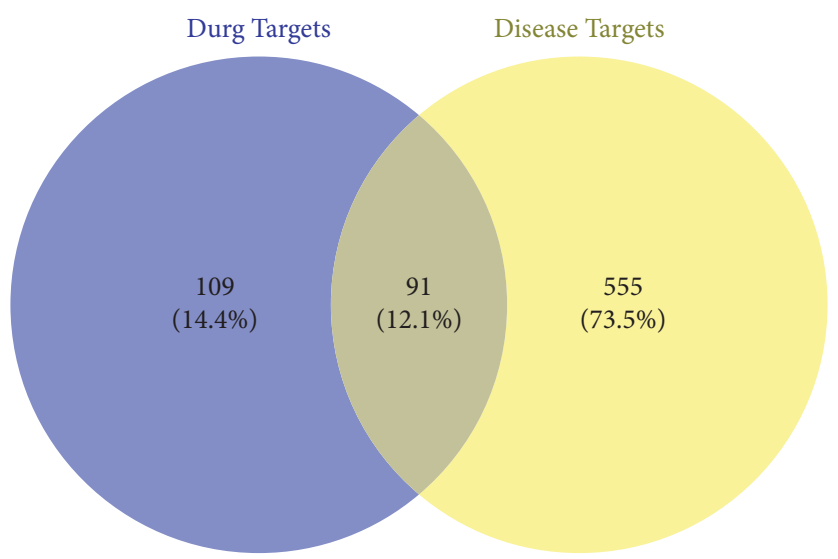

FIGURE 3: Intersection targets of AM and cisplatin-induced kidney damage. The blue zone indicates targets for active components of AM; the yellow zone indicates targets for cisplatin-induced kidney damage; and the overlap zone indicates the intersection targets.

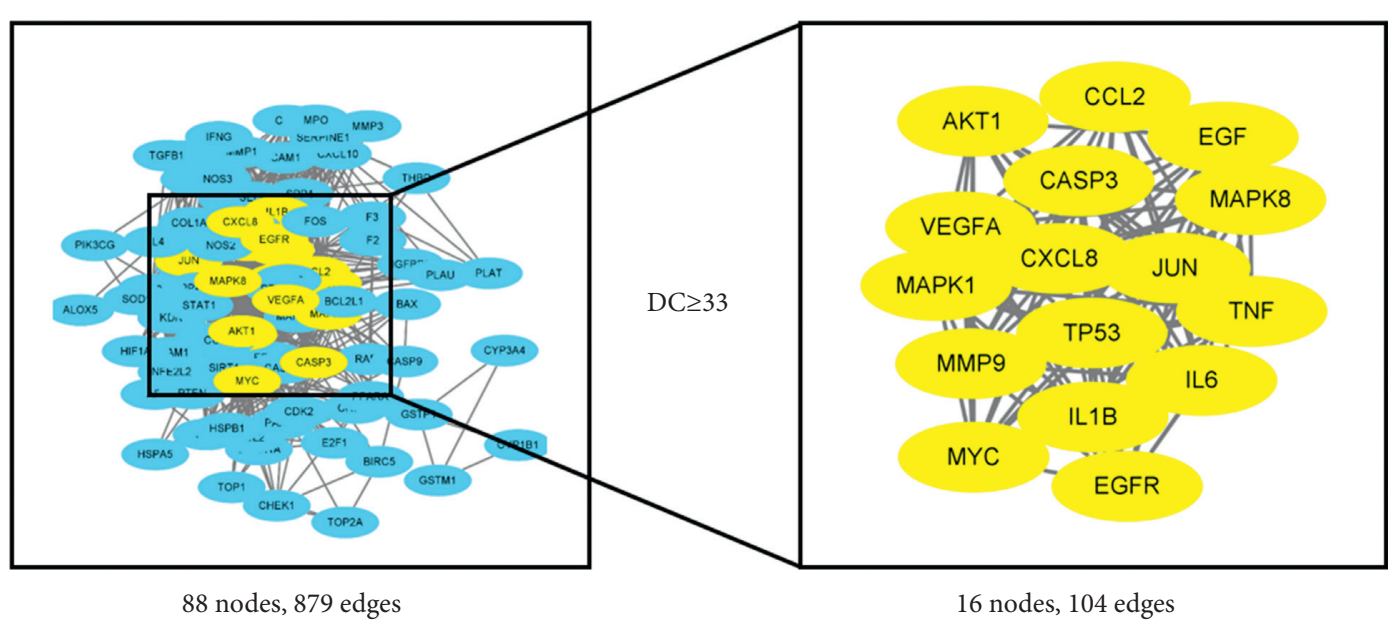

FIGURE 4: The process of filtering core targets. 16 core targets with DC $\geq 33$ (right panel) were selected from 91 intersection targets (left panel). Because 3 of the 91 common targets have no interaction with other targets with high confidence (confidence scores $>0.7$ ), there are only 88 nodes in the PPI network.

TABLE 3: The specific information of the 16 core targets.

\begin{tabular}{lcc}
\hline Gene symbol & Protein name & DC \\
\hline TP53 & Cellular tumor antigen p53 & 56 \\
AKT1 & RAC-alpha serine/threonine-protein kinase & 53 \\
IL6 & Interleukin-6 & 52 \\
TNF & Tumor necrosis factor-alpha & 50 \\
VEGFA & Vascular endothelial growth factor A & 47 \\
JUN & Transcription factor AP-1 & 46 \\
MAPK1 & Mitogen-activated protein kinase 1 & 45 \\
MAPK8 & Mitogen-activated protein kinase 8 \\
MMP9 & Matrix metalloproteinase-9 \\
EGF & Pro-epidermal growth factor \\
IL1B & Interleukin-1 beta \\
MYC & Myc proto-oncogene protein \\
EGFR & Epidermal growth factor receptor \\
CXCL8 & Interleukin-8 \\
CASP3 & Caspase-3 \\
CCL2 & C-C motif chemokine 2 \\
\hline
\end{tabular}




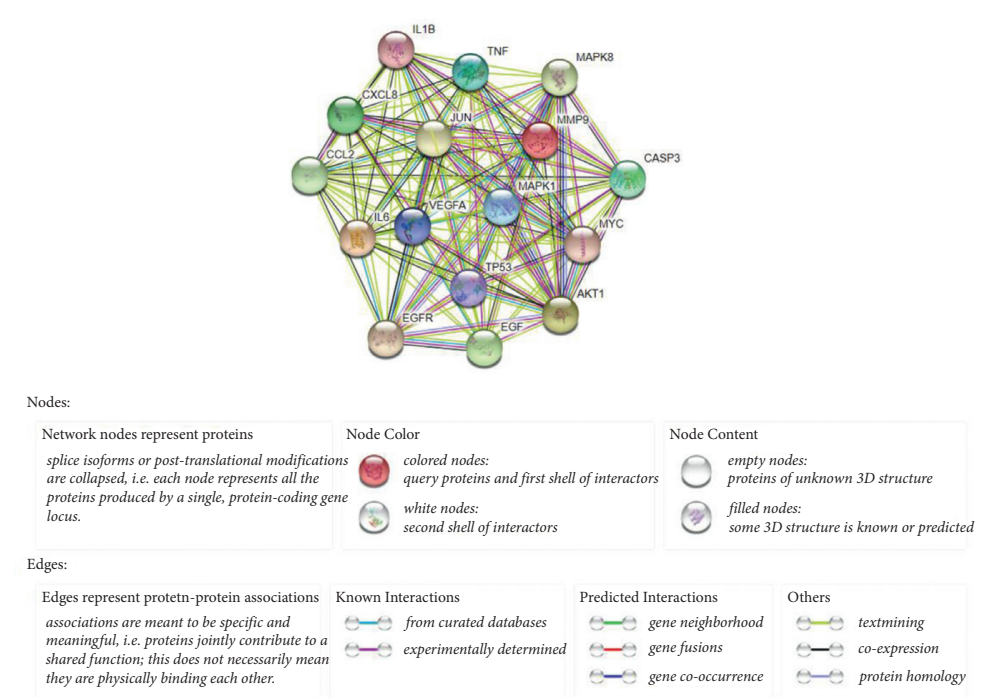

(a)

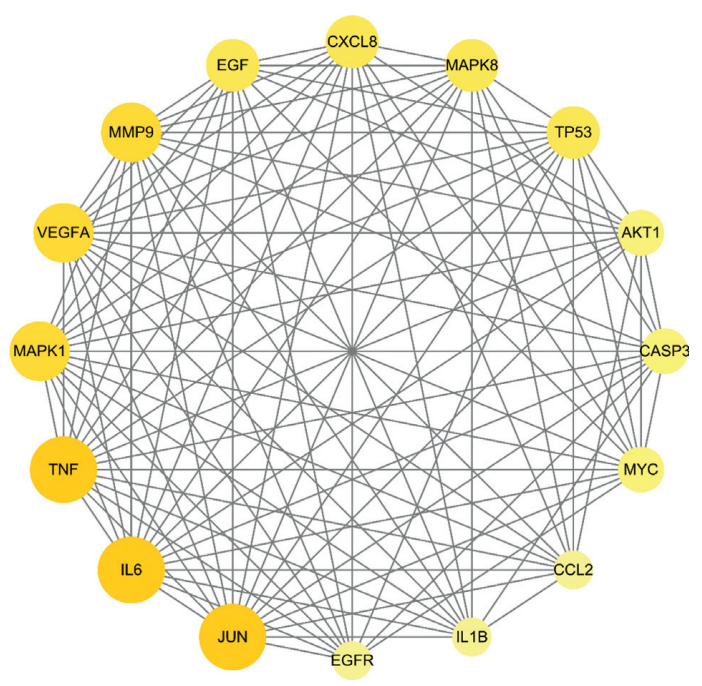

(b)

FIGURE 5: (a) The network from STRING platform directly. Nodes represent core targets; various edges mean different interactions. (b) The network was processed by Cytoscape 3.8.0. The interaction among the core targets is represented by the inner edge. The outer ring is arranged clockwise in descending order of significance of the nodes.

negative regulation of the apoptotic process, and so on. In the cell composition, the extracellular region is the main classification of these targets. Additionally, there were 23 terms enriched in the molecular function category, mainly including protein serine/threonine kinase activity, cytokine activity, transcription factor binding, growth factor activity, and so on. We selected the top 10 items according to the gene ratio, which are presented in Figure 6. The KEGG enrichment pathways analysis results revealed that there were 91 pathways in total, mainly include MAPK signaling pathway, PI3K-Akt signaling pathway, TNF signaling pathway, etc. We selected the top 20 pathways of KEGG according to the gene ratio, which are presented in Figure 7.

3.6. Active Component-Target-Pathway Analysis. For the aim at clarifying what the relationships between components, core targets, and pathways are, the significant pathways and biological processes, core targets, and active components of AM were used to build the C-T-P network (Figure 8). The targets related to pathways and biological processes are also listed in Table 4.

3.7. Molecular Docking. To identify potential drug-target interactions, we performed molecular docking to predict the binding affinities between the active components and core targets in the C-T-P network. Docking results are shown in Table 5. Herein, CDOCKER_ENERGY was used to evaluate the binding affinities. Lower binding energy represents a higher affinity between a ligand and a protein [34]. It can be seen that quercetin has a higher affinity with MAPK1, p53, matrix metalloproteinase-9 (MMP-9), epidermal growth factor receptor (EGFR), and caspase-3, suggesting that quercetin may mitigate cisplatin-induced kidney damage by binding to them.

\section{Discussion}

Cisplatin-induced kidney damage is a renal impairment caused by cisplatin, which is extremely harmful to human beings. It is reported that about $25-30 \%$ of patients developed symptoms of AKI after receiving cisplatin chemotherapy [4]. Besides hydration combined with mannitol diuretic, injection of AM or its active components is often used for treatment in the clinic [35-37]. Although the anticisplatin-induced kidney damage efficacy of AM has been demonstrated, the mechanism has not yet been clarified. Consequently, network pharmacology and molecular docking were performed to analyze the active components, targets, and related signaling pathways of AM against cisplatin-induced kidney damage and to figure out the potential mechanism of AM anti-cisplatin-induced kidney.

We obtained 20 active components and 200 corresponding targets from the TCMSP database and then constructed a component-target network, which mainly included quercetin, formononetin, isorhamnetin, and kaempferol. Modern pharmacological studies showed that quercetin has an anti-cisplatin-induced kidney injury effect, which is related to its antioxidant activity and the ability to inhibit kidney inflammation and renal tubular cell apoptosis [38]. In a recent study, it was reported that pretreatment with kaempferol reduced cisplatin-mediated oxidative stress, apoptosis, inflammation, kidney injury, as well as improved its function [39]. Formononetin, an O-methylated isoflavone, is one of the main bioactive ingredients in red clover plants. It was reported that formononetin can alleviate AKI induced by cisplatin and has many potential pharmacological effects, like anti-inflammatory, antioxidant, and antiapoptosis [40]. Isorhamnetin, a metabolite of quercetin, not only has anti-inflammatory and antioxidant effects but also enhances the anticancer effect of cisplatin [41, 42]. In 


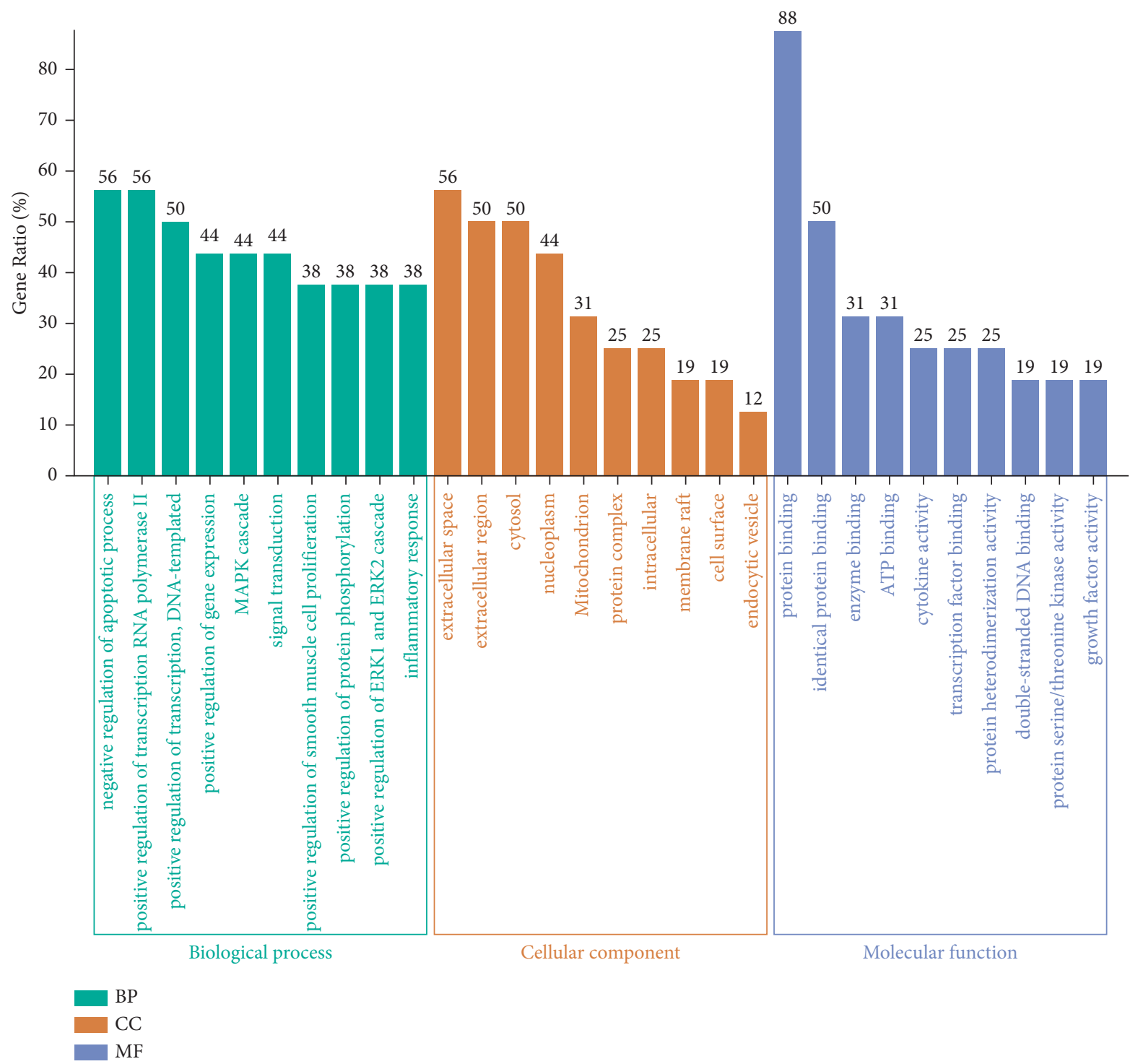

Figure 6: GO enrichment analysis. GO items and gene ratio are represented by the $x$-axis and $y$-axis, respectively.

addition, it is interesting that multiple AM active components can act on multiple different targets and have common pharmacological effects, which embodies the peculiarities of AM multicomponent, multitarget synergistic treatment.

We obtained 91 intersection targets between AM and cisplatin-induced kidney damage and further screened out 16 core targets of AM in the treatment of cisplatin-induced kidney damage. The targets are mainly associated with oxidative stress, apoptosis, inflammation, and cell proliferation. According to quantities of studies, apoptosis induced by TNF- $\alpha$, p53, and caspase-3 [43, 44] and inflammation induced by IL-6, TNF- $\alpha$, and interleukin-1 beta (IL-1 $\beta$ ) played an essential role in cisplatin-induced kidney damage $[45,46]$. It was reported that cisplatin increased the expression of proinflammatory cytokine TNF- $\alpha$, which induced an exogenous apoptotic pathway through its tumor necrosis factor receptor 1 (TNFR1) [47], and TNF- $\alpha$ can activate proinflammatory cytokines and chemokines such as NF- $\kappa \mathrm{B}$ and trigger oxidative stress, which ultimately aggravates kidney damage [48]. The study by $\mathrm{Xu}$ et al. also demonstrated that the expression of TNF- $\alpha$ further helped induce the expression of receptor-interacting protein 1 (RIP1), RIP3, and mixed lineage kinase domain-like protein (MLKL) in proximal tubular cells and enhanced the necrotic signaling pathway through positive feedback [49]. As mentioned in a literature review, the role of p53 in cisplatin-induced cytotoxicity mainly involved activation of the mitochondrial apoptotic pathway [47]. After exposure to cisplatin-induced cellular DNA damage, p53 was phosphorylated, and the proapoptotic protein Bax underwent structural modifications and mitochondrial membrane integrity changes, eventually downregulating the antiapoptotic proteins $\mathrm{Bcl}-2$ and $\mathrm{Bcl}-\mathrm{xL}$ and triggering the mitochondrial apoptotic pathway [50]. In addition, Yuan et al. proved that p53 promotes cisplatin-induced renal oxidative damage and apoptosis by regulating $\mathrm{P} 66 \mathrm{shc}$ and manganese-dependent superoxide dismutase (MnSOD) [51]. A recent study showed that the AMPK-p53-Bax signaling pathway played a crucial 


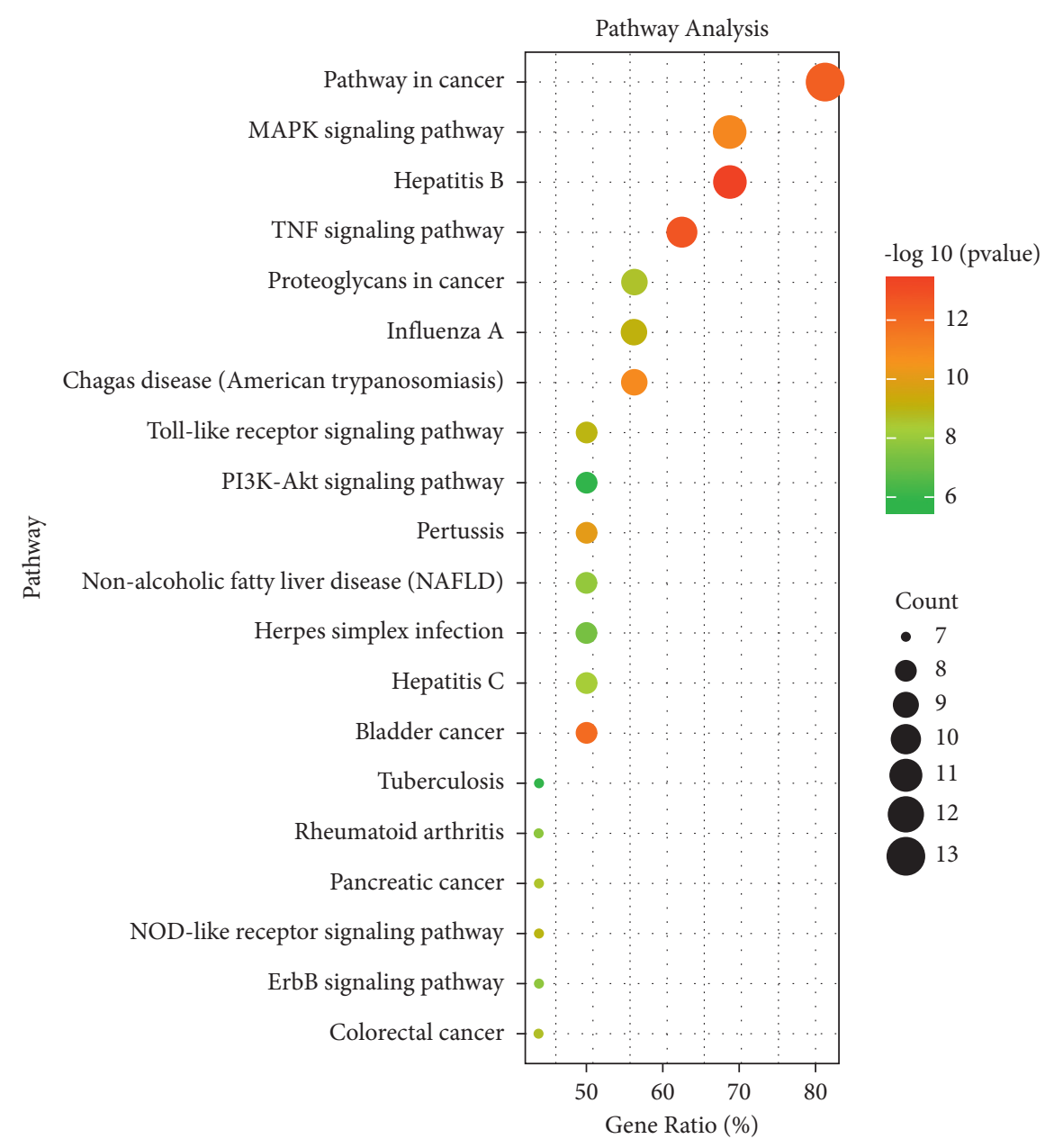

FIGURE 7: KEGG enrichment analysis. Gene ratio and pathways are represented by the $x$-axis and $y$-axis, respectively; the size and color of the dots indicate the gene count and the level of $P$ value, respectively.

role in cisplatin-induced apoptosis of renal tubular epithelial cells [44]. Many studies have shown that IL-6 is closely related to the inflammatory response in cisplatin-induced renal injury and cisplatin-induced inflammatory response can be alleviated by reducing the expression of IL-6 [52-55]. Another study showed that IL- 6 mediates the production and elimination of ROS in cisplatin-induced AKI [56]. All of the above-mentioned core targets and those not mentioned due to word limits are summarized in Table 6 .

Subsequently, GO and KEGG enrichment analyses were applied to 16 core targets. The result suggested that the PI3KAkt signaling pathway, MAPK signaling pathway, and Tolllike receptor signaling pathway were suggested to have a significant role in the treatment of cisplatin-induced kidney damage by AM. It has been reported that the MAPK pathway is central to the regulation of inflammation and oxidative stress in cisplatin renal injury and that TNF- $\alpha$ can activate the MAPK pathway [65]. Besides, Ning et al. found the PI3K-Akt signaling pathway plays an essential role in defending renal tubular epithelial cells from apoptosis induced by cisplatin [66]. Meanwhile, many studies have demonstrated that quercetin can suppress the formation of reactive oxygen species, activate the JNK/P38 MAPK signaling pathway, and regulate the PI3K-Akt pathway [67-69]. Therefore, quercetin may reduce oxidative stress by regulating the MAPK pathway and PI3K-Akt pathway in the treatment of cisplatin-induced kidney. The results of molecular docking suggested that the most likely key targets are MAPK1, TP53, and EGFR. In addition, kaempferol could block the MAPK cascade and reduce the expression of JNK, TNF- $\alpha$, and ERK1/2, thus improving cisplatin-induced renal injury [39, 70]. What is more, the Toll-like receptor signaling pathway can activate the releasing of downstream cytokines such as TNF- $\alpha$ and IL- $1 \beta$, leading to increased inflammatory cell infiltration and inflammatory responses in renal tissue [71, 72]. Meanwhile, a study has shown that kaempferol dramatically suppresses the upregulation of toll-like receptor 4, blocking the Toll-like receptor signaling pathway to reduce the inflammatory response [70]. Molecular docking suggested that quercetin most likely affected some pathways by combining with p53, MAPK, MMP-9, EGFR, and caspase- 3 and then finally achieved the effect of reducing cisplatin-induced renal damage. The pathways discussed above are shown in Figure 9.

In the C-T-P network, each pathway corresponds to a plurality of targets, and each target is connected with a plurality of pathways that are connected with each other 


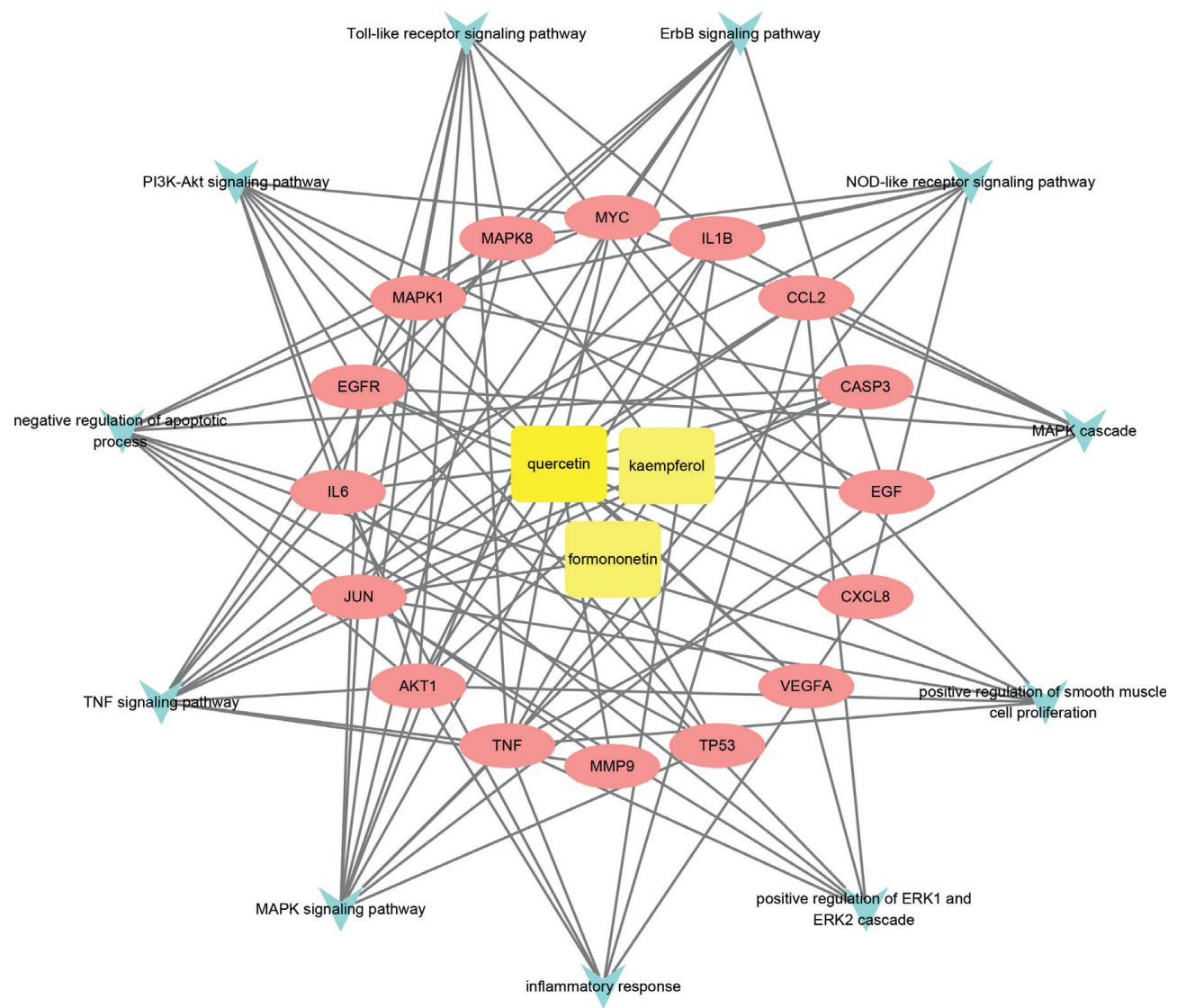

Figure 8: The C-T-P network of AM against cisplatin-induced kidney damage. The significant compounds, core targets, and pathways are represented by the yellow rectangular nodes, pink oval nodes, and arrow nodes, respectively.

TABLE 4: Information of related target gene symbols.

\begin{tabular}{lc}
\hline Pathway or biological process & Gene symbol of related target \\
\hline MAPK signaling pathway & JUN, MAPK8, EGF, IL1B, MYC, CASP3, MAPK1, AKT1, TNF, TP53, EGFR \\
TNF signaling pathway & IL6, JUN, MAPK8, IL1B, CASP3, MAPK1, CCL2, AKT1, TNF, MMP9 \\
Toll-like receptor signaling pathway & IL6, JUN, MAPK8, CXCL8, IL1B, MAPK1, AKT1, TNF \\
Positive regulation of smooth muscle cell proliferation & IL6, JUN, MYC, AKT1, TNF, EGFR \\
PI3K-Akt signaling pathway & IL6, EGF, MYC, MAPK1, AKT1, TP53, EGFR, VEGFA \\
NOD-like receptor signaling pathway & IL6, MAPK8, CXCL8, IL1B, MAPK1, CCL2, TNF \\
ErbB signaling pathway & JUN, MAPK8, EGF, MYC, MAPK1, AKT1, EGFR \\
Negative regulation of apoptotic process & IL6, MAPK8, MYC, CASP3, AKT1, TP53, MMP9, EGFR, VEGFA \\
MAPK cascade & IL6, JUN, CCL2, TNF, EGFR, VEGFA \\
Positive regulation of ERK1 and ERK2 cascade & IL6, CXCL8, IL1B, CCL2, AKT1, TNF \\
Inflammatory response &
\end{tabular}

TABLE 5: The results of molecular docking.

\begin{tabular}{lccc}
\hline Component & Target (gene symbol/protein name) & PDB ID & CDOCKER_ENERGY (kcal/mol) \\
\hline Quercetin & TP53/cellular tumor antigen p53 & $4 \mathrm{BUZ}$ & -39.4732 \\
Quercetin & AKT1/RAC-alpha serine/threonine-protein kinase & 1H10 & -26.3244 \\
Quercetin & IL6/interleukin-6 & 1ALU & -26.6531 \\
Quercetin & TNF/tumor necrosis factor alpha & 3IT8 & -15.4083 \\
Quercetin & VEGFA/vascular endothelial growth factor A & $4 \mathrm{GLU}$ & -24.2995 \\
Quercetin & JUN/transcription factor AP-1 & 5FV8 & -26.5148 \\
Quercetin & MAPK1/mitogen-activated protein kinase 1 & $2 \mathrm{WAJ}$ & -37.9661 \\
Quercetin & MMP9/matrix metalloproteinase-9 & -39.5964 \\
Quercetin & EGF/pro-epidermal growth factor & 3NJP & -14.2012 \\
Quercetin & IL1B/interleukin-1 $\beta$ & 1IRA & -22.1756 \\
Quercetin & MYC/Myc proto-oncogene protein & $4 \mathrm{Y} 7 \mathrm{R}$ & -26.1611 \\
\hline
\end{tabular}


TABle 5: Continued.

\begin{tabular}{lccc}
\hline Component & Target (gene symbol/protein name) & PDB ID & CDOCKER_ENERGY (kcal/mol) \\
\hline Quercetin & EGFR/epidermal growth factor receptor & 2JIV & -40.9744 \\
Quercetin & CXCL8/interleukin-8 & 6LFM & -24.7365 \\
Quercetin & CASP3/caspase-3 & 3DEI & -33.4638 \\
Quercetin & CCL2/C-C motif chemokine 2 & 4 DN4 & -32.0911 \\
Formononetin & JUN/transcription factor AP-1 & $5 \mathrm{FV} 8$ & -9.6172 \\
Kaempferol & AKT1/RAC-alpha serine/threonine-protein kinase & $1 \mathrm{H} 10$ & -21.7786 \\
Kaempferol & TNF/tumor necrosis factor-alpha & -8.2117 \\
Kaempferol & JUN/transcription factor AP-1 & 3FT8 & -20.2562 \\
Kaempferol & MAPK8/mitogen-activated protein kinase 8 & 1UKI & -26.8723 \\
Kaempferol & CASP3/caspase-3 & 3DEI & -24.6836 \\
\hline
\end{tabular}

TABLE 6: Summary of key targets' functional research.

\begin{tabular}{|c|c|c|}
\hline Key target & Functional research in cisplatin-induced kidney injury & References \\
\hline TNF- $\alpha$ & $\begin{array}{l}\text { 1. Cisplatin } \longrightarrow \text { TNF- } \alpha \longrightarrow \text { TNFR } 1 \longrightarrow \text { exogenous apoptotic pathway } \\
\text { 2. Cisplatin } \longrightarrow \text { TNF- } \alpha \longrightarrow \text { NF- } \kappa \mathrm{B} \longrightarrow \text { oxidative stress } \longrightarrow \text { kidney damage } \\
\text { 3. TNF- } \alpha \longrightarrow \text { RIP1, RIP3, and MLKL } \longrightarrow \text { necrotic signaling pathway }\end{array}$ & $\begin{array}{l}47] \\
{[48]} \\
{[49]}\end{array}$ \\
\hline p53 & $\begin{array}{l}\text { 1. Cisplatin } \longrightarrow \text { DNA damage } \longrightarrow \mathrm{p}-\mathrm{p} 53 \longrightarrow \mathrm{Bax} \longrightarrow \mathrm{Bcl}-2 \text { and } \mathrm{Bcl}-\mathrm{xL} \longrightarrow \text { mitochondrial apoptotic } \\
\text { pathway } \\
\text { 2. Cisplatin } \longrightarrow \mathrm{p} 53 \longrightarrow \text { P66shc and MnSOD } \longrightarrow \text { oxidative damage and apoptosis } \\
\text { 3. AMPK-p53-Bax signaling pathway (Cisplatin } \longrightarrow \text { AMPK } \longrightarrow \text { p-p53 } \longrightarrow \text { Bax and caspase } \\
\text { 3 } \longrightarrow \text { apoptosis) }\end{array}$ & $\begin{array}{l}{[47]} \\
{[51]} \\
{[44]}\end{array}$ \\
\hline IL-6 & $\begin{array}{l}\text { 1. Cisplatin } \longrightarrow \text { IL- } 6 \longrightarrow \text { inflammatory response } \longrightarrow \text { cisplatin-induced kidney injury } \\
\text { 2. IL- } 6 \text { mediates the production and elimination of ROS in cisplatin-induced AKI }\end{array}$ & $\begin{array}{l}{[52-55]} \\
{[56]}\end{array}$ \\
\hline VEGFA & VEGFA $\longrightarrow$ improve microcirculation and antiapoptotic $\longrightarrow$ against cisplatin-induced AKI & [57] \\
\hline EGF/EGFR & EGF-EGFR $\longrightarrow$ tyrosine kinase $\longrightarrow$ Ras $\longrightarrow$ MAPK signaling pathway & [58] \\
\hline $\begin{array}{l}\text { MAPK1/MAPK8/ } \\
\text { JUN }\end{array}$ & MAPK signaling pathway & {$[54,59-61]$} \\
\hline IL-1 $\beta$ & IL- $1 \beta$ and IL- $6 \longrightarrow$ neutrophil infiltration $\longrightarrow$ cisplatin-induced AKI & [62] \\
\hline CXCL8 & $\begin{array}{c}\text { Cisplatin } \longrightarrow \text { CXCL8 } \longrightarrow \text { neutrophil recruitment } \underset{\text { kidney injury }}{\longrightarrow} \text { inflammatory response } \longrightarrow \text { cisplatin-induced } \\
\text {. }\end{array}$ & {$[63,64]$} \\
\hline Caspase-3 & Cisplatin $\longrightarrow$ TNFR1, TNFR2, and FasR $\longrightarrow$ caspase- $8 \longrightarrow$ caspase- $3 \longrightarrow$ apoptosis & [43] \\
\hline
\end{tabular}

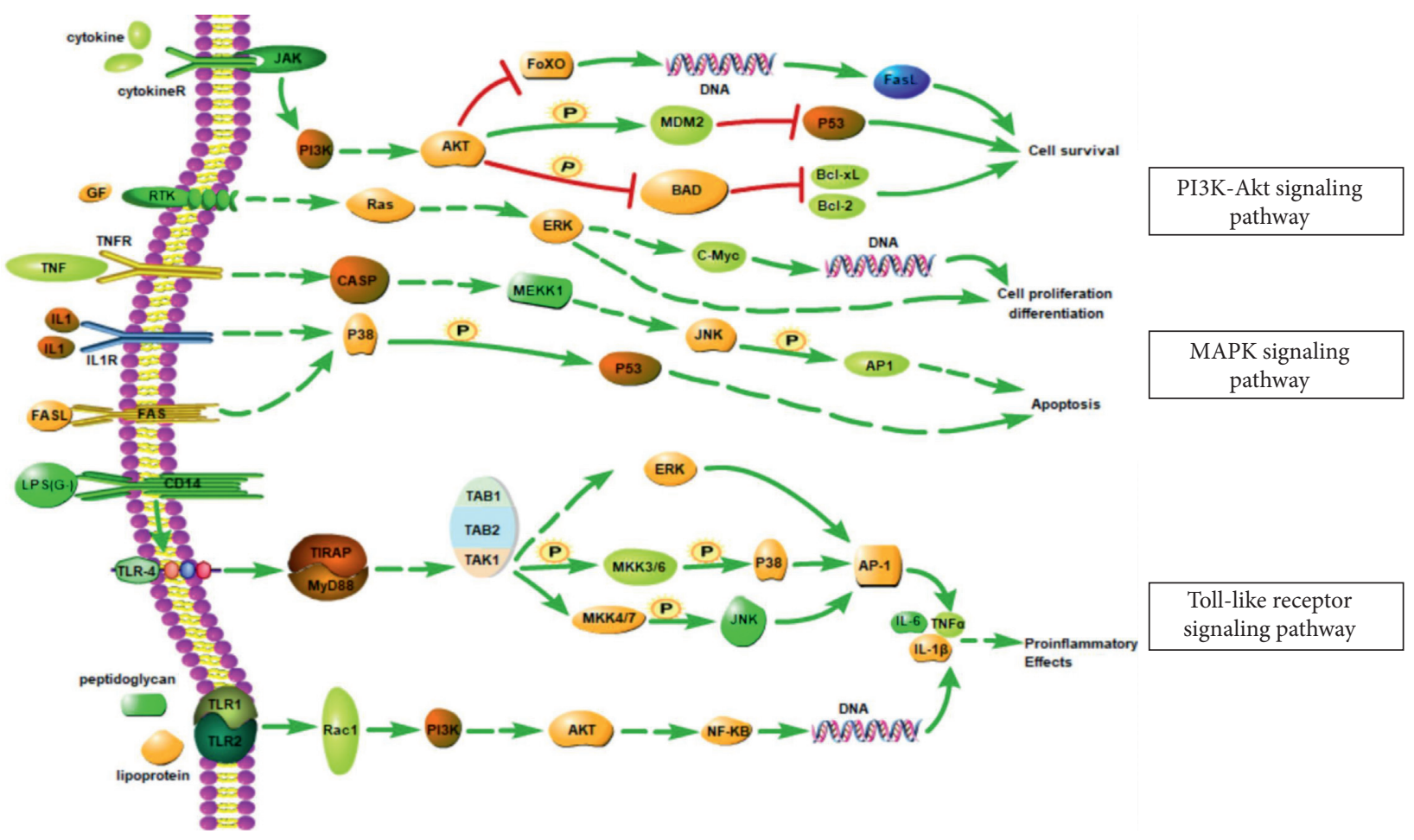

Figure 9: Key biological pathways. 
through a common target. It was suggested that AM synergistically treats cisplatin-induced kidney damage through a multicomponent, multitarget, and multipathway mechanism.

\section{Conclusions}

In summary, the present research aimed to figure out the molecular mechanism of AM against cisplatin-induced kidney damage by network pharmacology and molecular docking. Screening of active ingredients and molecular docking studies showed that quercetin, kaempferol, and formononetin were significant active ingredients to AM against cisplatin-induced kidney damage. The C-T-P network suggested that the MAPK pathway, PI3K-Akt signaling pathway, and Toll-like receptor signaling pathway had important roles in AM treatment of cisplatin-induced kidney damage. In addition, one of the significant findings is that AM treats cisplatin-induced kidney injury to multicomponent, multitarget, and multipathway synergistic effects. Unfortunately, this study has no corresponding experimental validation that is what we need to do in the future. In a nutshell, this study provided a rationale for further research of cisplatin-induced renal injury and a new orientation of development and application of AM.

\section{Abbreviations}

$\begin{array}{ll}\text { AM: } & \text { Astragalus membranaceus } \\ \text { AKI: } & \text { Acute kidney injury } \\ \text { C-T-P } & \text { Network of active component-target- } \\ \text { network: } & \text { pathway } \\ \text { DC: } & \text { Degree centrality } \\ \text { DL: } & \text { Drug likeness } \\ \text { DS: } & \text { Discovery Studio } \\ \text { EGFR: } & \text { Epidermal growth factor receptor } \\ \text { GO: } & \text { Gene Ontology } \\ \text { IL-1 } \beta: & \text { Interleukin-1 } \beta \\ \text { IL-6: } & \text { Interleukin-6 } \\ \text { KEGG: } & \text { Kyoto Encyclopedia of Genes and Genomes } \\ \text { MAPK1: } & \text { Mitogen-activated protein kinase 1 } \\ \text { MLKL: } & \text { Mixed lineage kinase domain-like protein } \\ \text { MMP-9: } & \text { Matrix metalloproteinase-9 } \\ \text { MnSOD: } & \text { Manganese-dependent superoxide dismutase } \\ \text { OB: } & \text { Oral bioavailability } \\ \text { p53: } & \text { Cellular tumor antigen p53 } \\ \text { PDB: } & \text { Protein Data Bank } \\ \text { PPI: } & \text { Protein-protein interaction } \\ \text { RIP1: } & \text { Receptor-interacting protein 1 } \\ \text { TCMSP: } & \text { Traditional Chinese Medicine Systems } \\ & \text { Pharmacology } \\ \text { TNFR1: } & \text { Tumor necrosis factor receptor 1 } \\ \text { TNF- } \alpha: & \text { Tumor necrosis factor-alpha } \\ \text { VEGFA: } & \text { Vascular endothelial growth factor A. } \\ & \end{array}$

\section{Data Availability}

Processed data are contained within the article. Raw data are available from the corresponding author upon request.

\section{Conflicts of Interest}

The authors declare that there are no conflicts of interest.

\section{Authors' Contributions}

Congchao Jia conceived and mainly performed the research; Pengyu Wang collected and collated data on drug and disease; Binyou Wang and Yiwei Wang carried out the molecular docking analysis; Congchao Jia and Rong Chen drafted the manuscript; and Xianchao Pan revised the manuscript. All authors read and approved the final manuscript.

\section{Acknowledgments}

The authors greatly appreciate the financial support from the Natural Science Project of Sichuan Provincial Health Department (no. 110327) and Natural Science Project of Luzhou Science and Technology Bureau (no. 30178).

\section{References}

[1] A. Ozkok and C. L. Edelstein, "Pathophysiology of cisplatininduced acute kidney injury," BioMed Research International, vol. 2014, Article ID 967826, 17 pages, 2014.

[2] K. M. E. Maedeh, S. E. Alavi, S. Shahbazian, and H. Ebrahimi Shahmabadi, "Drug delivery of cisplatin to breast cancer by polybutylcyanoacrylate nanoparticles," Advances in Polymer Technology, vol. 37, 2016.

[3] S. Latcha, E. A. Jaimes, S. Patil, I. G. Glezerman, S. Mehta, and C. D. Flombaum, "Long-term renal outcomes after cisplatin treatment," Clinical Journal of the American Society of $\mathrm{Ne}$ phrology, vol. 11, no. 7, pp. 1173-1179, 2016.

[4] J. Y. Kim, J. Jo, K. Kim et al., "Pharmacological activation of Sirt1 ameliorates cisplatin-induced acute kidney injury by suppressing apoptosis, oxidative stress, and inflammation in mice," Antioxidants, vol. 8, no. 8, 2019.

[5] Z. K. Zsengellér, L. Ellezian, D. Brown et al., "Cisplatin nephrotoxicity involves mitochondrial injury with impaired tubular mitochondrial enzyme activity," Journal of Histochemistry and Cytochemistry, vol. 60, no. 7, pp. 521-529, 2012.

[6] M. Ichinomiya, A. Shimada, N. Ohta et al., "Demonstration of mitochondrial damage and mitophagy in cisplatin-mediated nephrotoxicity," Tohoku Journal of Experimental Medicine, vol. 246, no. 1, pp. 1-8, 2018.

[7] K. Tanabe, Y. Tamura, M. A. Lanaspa et al., "Epicatechin limits renal injury by mitochondrial protection in cisplatin nephropathy," American Journal of Physiology-Renal Physiology, vol. 303, no. 9, pp. F1264-F1274, 2012.

[8] Y. Yang, H. Liu, F. Liu, and Z. Dong, "Mitochondrial dysregulation and protection in cisplatin nephrotoxicity," Archives of Toxicology, vol. 88, no. 6, pp. 1249-1256, 2014.

[9] C. Brooks, Q. Wei, S.-G. Cho, and Z. Dong, "Regulation of mitochondrial dynamics in acute kidney injury in cell culture and rodent models," Journal of Clinical Investigation, vol. 119, no. 5, pp. 1275-1285, 2009.

[10] S. Manohar and N. Leung, "Cisplatin nephrotoxicity: a review of the literature," Journal of Nephrology, vol. 31, no. 1, pp. 15-25, 2018.

[11] N. Pabla and Z. Dong, "Cisplatin nephrotoxicity: mechanisms and renoprotective strategies," Kidney International, vol. 73, no. 9, pp. 994-1007, 2008. 
[12] Y. Liang, Q. Zhang, L. Zhang, R. Wang, X. Xu, and X. Hu, "Astragalus membranaceus treatment protects Raw264.7 cells from influenza virus by regulating G1 phase and the TLR3mediated signaling pathway, evidence-based complementary and alternative medicine," Evidence-Based Complementary and Alternative Medicine, vol. 2019, Article ID 2971604, 10 pages, 2019.

[13] C. Cheon and S. G. Ko, "A phase I study to evaluate the safety of the herbal medicine SH003 in patients with solid cancer," Integrative Cancer Therapies, vol. 19, 2020.

[14] M. Shahzad, A. Shabbir, K. Wojcikowski, H. Wohlmuth, and G. C. Gobe, "The antioxidant effects of radix astragali (Astragalus membranaceus and related species) in protecting tissues from injury and disease," Current Drug Targets, vol. 17, no. 12, pp. 1331-1340, 2016.

[15] S. Li, Y. Sun, J. Huang et al., "Anti-tumor effects and mechanisms of Astragalus membranaceus (AM) and its specific immunopotentiation: status and prospect," Journal of Ethnopharmacology, vol. 258, Article ID 112797, 2020.

[16] X. Tang, H. Wang, H. Chen, S. Sun, H. Chen, and R. Pan, "Protective effects of astragalus membranaceus and ligustrazine on rat brain microvascular endothelial cell injury after oxygen-glucose depri-vation/reoxygenation by supressing the PKC $\delta / M A R C K S$ pathway," Combinatorial Chemistry \& High Throughput Screening, vol. 24, 2020.

[17] Q. Ma, Y. Xu, L. Tang et al., “Astragalus polysaccharide attenuates cisplatin-induced acute kidney injury by suppressing oxidative damage and mitochondrial dysfunction," BioMed Research International, vol. 2020, Article ID 2851349, 12 pages, 2020.

[18] A. Cao, H. He, Q. Wang, L. Li, Y. An, and X. Zhou, "Evidence of Astragalus injection combined platinum-based chemotherapy in advanced nonsmall cell lung cancer patients," Medicine, vol. 98, no. 11, Article ID e14798, 2019.

[19] R.-Z. Zhang, S.-J. Yu, H. Bai, and K. Ning, "TCM-Mesh: the database and analytical system for network pharmacology analysis for TCM preparations," Scientific Reports, vol. 7, no. 1, p. 2821, 2017.

[20] Z. Zhou, B. Chen, S. Chen et al., "Applications of network pharmacology in traditional Chinese medicine research, evidence-based complementary and alternative medicine," $E v$ idence-Based Complementary and Alternative Medicine, vol. 2020, Article ID 1646905, 7 pages, 2020.

[21] C. Duan, Y. Li, X. Dong, W. Xu, and Y. Ma, "Network pharmacology and reverse molecular docking-based prediction of the molecular targets and pathways for avicularin against cancer," Combinatorial Chemistry \& High Throughput Screening, vol. 22, no. 1, pp. 4-12, 2019.

[22] J. Ru, P. Li, J. Wang et al., "TCMSP: a database of systems pharmacology for drug discovery from herbal medicines," Journal of Cheminformatics, vol. 6, no. 1, p. 13, 2014.

[23] C. Zheng, T. Pei, C. Huang et al., "A novel systems pharmacology platform to dissect action mechanisms of traditional Chinese medicines for bovine viral diarrhea disease," European Journal of Pharmaceutical Sciences, vol. 94, pp. 33-45, 2016.

[24] H. Geng, X. Chen, and C. Wang, "Systematic elucidation of the pharmacological mechanisms of Rhynchophylline for treating epilepsy via network pharmacology," BMC complementary medicine and therapies, vol. 21, no. 1, p. 9, 2021.

[25] T. Pei, C. Zheng, C. Huang et al., "Systematic understanding the mechanisms of vitiligo pathogenesis and its treatment by Qubaibabuqi formula," Journal of Ethnopharmacology, vol. 190, pp. 272-287, 2016.
[26] M. Safran, I. Dalah, J. Alexander et al., "GeneCards Version 3: the human gene integrator," Database, vol. 2010, Article ID baq020, 2010.

[27] J. S. Amberger, C. A. Bocchini, F. Schiettecatte, A. F. Scott, and A. Hamosh, "OMIM.org: online Mendelian Inheritance in Man (OMIM $\left.{ }^{\circledR}\right)$, an online catalog of human genes and genetic disorders," Nucleic Acids Research, vol. 43, pp. D789-D798, 2015.

[28] J. S. Amberger and A. Hamosh, "Searching online mendelian inheritance in man (OMIM): a knowledgebase of human genes and genetic phenotypes," Current protocols in bioinformatics, vol. 58, pp. 1-12, 2017.

[29] D. Szklarczyk, A. L. Gable, D. Lyon et al., "STRING v11: protein-protein association networks with increased coverage, supporting functional discovery in genome-wide experimental datasets," Nucleic Acids Research, vol. 47, pp. D607d613, 2019.

[30] M. Legeay, N. T. Doncheva, J. H. Morris, and L. J. Jensen, "Visualize omics data on networks with omics visualizer, a Cytoscape app," A Cytoscape Application, vol. 9, p. 157, 2020.

[31] D. W. Huang, B. T. Sherman, and R. A. Lempicki, "Systematic and integrative analysis of large gene lists using DAVID bioinformatics resources," Nature Protocols, vol. 4, no. 1, pp. 44-57, 2009.

[32] M. Dedhia, K. Kohetuk, W. E. Crusio, and A. Delprato, "Introducing high school students to the Gene Ontology classification system," F1000Research, vol. 8, p. 241, 2019.

[33] Y. W. Burenbatu, W. Narisu, E. Hashengaowa, and H. B. Guihua, "iTRAQ-based quantitative proteomics analysis of immune thrombocytopenia patients before and after Qishunbaolier treatment," Rapid Communications in Mass Spectrometry: Rapid Communications in Mass Spectrometry, vol. 35, no. 3, Article ID e8993, 2021.

[34] Y. Quah, S. J. Lee, E. B. Lee et al., "Cornus officinalis ethanolic extract with potential anti-allergic, anti-inflammatory, and antioxidant activities," Nutrients, vol. 12, no. 11, 2020.

[35] P. Duan and Z. M. Wang, "Clinical study on effect of Astragalus in efficacy enhancing and toxicity reducing of chemotherapy in patients of malignant tumor," Chinese journal of integrated traditional and Western medicine, vol. 22, no. 7, pp. 515-517, 2002.

[36] L. Guo, S.-P. Bai, L. Zhao, and X.-H. Wang, "Astragalus polysaccharide injection integrated with vinorelbine and cisplatin for patients with advanced non-small cell lung cancer: effects on quality of life and survival," Medical Oncology, vol. 29, no. 3, pp. 1656-1662, 2012.

[37] Y. H. Zou and X. M. Liu, "Effect of astragalus injection combined with chemotherapy on quality of life in patients with advanced non-small cell lung cancer," Chinese journal of integrated traditional and Western medicine, vol. 23, no. 10, pp. 733-735, 2003.

[38] M. Najafi, S. Tavakol, A. Zarrabi, and M. Ashrafizadeh, "Dual role of quercetin in enhancing the efficacy of cisplatin in chemotherapy and protection against its side effects: a review," Archives of Physiology and Biochemistry, vol. 16, pp. 1-15, 2020.

[39] Z. Wang, W. Sun, X. Sun, Y. Wang, and M. Zhou, "Kaempferol ameliorates Cisplatin induced nephrotoxicity by modulating oxidative stress, inflammation and apoptosis via ERK and NF- $\kappa$ B pathways," AMB Express, vol. 10, no. 1, p. 58, 2020.

[40] Y. Hao, J. Miao, W. Liu, L. Peng, Y. Chen, and Q. Zhong, "Formononetin protects against cisplatin-induced acute kidney injury through activation of the PPAR $\alpha / \mathrm{Nrf} 2 / \mathrm{HO}-1 /$ 
NQO1 pathway," International Journal of Molecular Medicine, vol. 47, no. 2, pp. 511-522, 2021.

[41] G. Gong, Y.-Y. Guan, Z.-L. Zhang et al., "Isorhamnetin: a review of pharmacological effects," Biomedicine \& Pharmacotherapy, vol. 128, Article ID 110301, 2020.

[42] B. Y. Zhang, Y. M. Wang, H. Gong et al., "Isorhamnetin flavonoid synergistically enhances the anticancer activity and apoptosis induction by cis-platin and carboplatin in nonsmall cell lung carcinoma (NSCLC)," International Journal of Clinical and Experimental Pathology, vol. 8, no. 1, pp. 25-37, 2015.

[43] K. Tsuruya, T. Ninomiya, M. Tokumoto et al., "Direct involvement of the receptor-mediated apoptotic pathways in cisplatin-induced renal tubular cell death," Kidney International, vol. 63, no. 1, pp. 72-82, 2003.

[44] X. Jin, C. An, B. Jiao, R. L. Safirstein, and Y. Wang, “AMPactivated protein kinase contributes to cisplatin-induced renal epithelial cell apoptosis and acute kidney injury," American Journal of Physiology - Renal Physiology, vol. 319, no. 6, pp. F1073-f1080, 2020.

[45] J. Y. Kim, J. Jo, J. Leem, and K. K. Park, "Inhibition of p300 by garcinol protects against cisplatin-induced acute kidney injury through suppression of oxidative stress, inflammation, and tubular cell death in mice," Antioxidants, vol. 9, no. 12, 2020.

[46] P. Mukhopadhyay, M. Rajesh, H. Pan et al., "Cannabinoid-2 receptor limits inflammation, oxidative/nitrosative stress, and cell death in nephropathy," Free Radical Biology and Medicine, vol. 48, no. 3, pp. 457-467, 2010.

[47] C. Y. Fang, D. Y. Lou, L. Q. Zhou et al., "Natural products: potential treatments for cisplatin-induced nephrotoxicity," Acta Pharmacologica Sinica, vol. 4, 2021.

[48] K. R. McSweeney, L. K. Gadanec, T. Qaradakhi, B. A. Ali, A. Zulli, and V. Apostolopoulos, "Mechanisms of cisplatininduced acute kidney injury: pathological mechanisms," Pharmacological Interventions, and Genetic Mitigations, Cancers, vol. 13, no. 7, 2021.

[49] Y. Xu, H. Ma, J. Shao et al., "A role for tubular necroptosis in cisplatin-induced AKI," Journal of the American Society of Nephrology, vol. 26, no. 11, pp. 2647-2658, 2015.

[50] H. Servais, A. Ortiz, O. Devuyst, S. Denamur, P. M. Tulkens, and M.-P. Mingeot-Leclercq, "Renal cell apoptosis induced by nephrotoxic drugs: cellular and molecular mechanisms and potential approaches to modulation," Apoptosis, vol. 13, no. 1, pp. 11-32, 2008.

[51] Y. Yuan, H. Wang, Y. Wu et al., "P53 contributes to cisplatin induced renal oxidative damage via regulating P66shc and MnSOD," Cellular Physiology and Biochemistry, vol. 37, no. 4, pp. 1240-1256, 2015.

[52] W. Wasyluk and A. Zwolak, "Metabolic alterations in sepsis," Journal of Clinical Medicine, vol. 10, no. 11, 2021.

[53] S. Liu, X. Zhang, and J. Wang, "Isovitexin protects against cisplatin-induced kidney injury in mice through inhibiting inflammatory and oxidative responses," International Immunopharmacology, vol. 83, Article ID 106437, 2020.

[54] J. S. Deng, W. P. Jiang, C. C. Chen et al., "Cordyceps cicadae mycelia ameliorate cisplatin-induced acute kidney injury by suppressing the TLR4/NF- $\kappa \mathrm{B} / \mathrm{MAPK}$ and activating the $\mathrm{HO}$ $1 / \mathrm{Nrf} 2$ and sirt-1/AMPK pathways in mice," Oxidative medicine and cellular longevity, vol. 2020, Article ID 7912763, 2020.

[55] M. Al Fayi, H. Otifi, M. Alshyarba, A. A. Dera, and P. Rajagopalan, "Thymoquinone and curcumin combination protects cisplatin-induced kidney injury, nephrotoxicity by attenuating NF $\kappa \mathrm{B}, \mathrm{KIM}-1$ and ameliorating Nrf2/HO-1 signalling," Journal of Drug Targeting, vol. 28, no. 9, pp. 913-922, 2020.

[56] S. Mitazaki, M. Hashimoto, Y. Matsuhashi et al., "Interleukin6 modulates oxidative stress produced during the development of cisplatin nephrotoxicity," Life Sciences, vol. 92, no. 12, pp. 694-700, 2013.

[57] L. Yuan, M.-J. Wu, H.-Y. Sun et al., "VEGF-modified human embryonic mesenchymal stem cell implantation enhances protection against cisplatin-induced acute kidney injury," American Journal of Physiology-Renal Physiology, vol. 300, no. 1, pp. F207-F218, 2011.

[58] P. S. Randhawa, N. A. Farasati, Y. Huang, M. Y. Mapara, and R. Shapiro, "Viral drug sensitivity testing using quantitative PCR: effect of tyrosine kinase inhibitors on polyomavirus BK replication," American Journal of Clinical Pathology, vol. 134, no. 6, pp. 916-920, 2010.

[59] X. Ma, C. Dang, H. Kang et al., "Saikosaponin-D reduces cisplatin-induced nephrotoxicity by repressing ROS-mediated activation of MAPK and NF- $\kappa$ B signalling pathways," International Immunopharmacology, vol. 28, no. 1, pp. 399408, 2015.

[60] A. Tomar, S. Kaushik, S. I. Khan et al., "The dietary isoflavone daidzein mitigates oxidative stress, apoptosis, and inflammation in CDDP-induced kidney injury in rats: impact of the MAPK signaling pathway," Journal of Biochemical and Molecular Toxicology, vol. 34, no. 2, Article ID e22431, 2020.

[61] A. K. Sahu, V. K. Verma, E. Mutneja et al., "Mangiferin attenuates cisplatin-induced acute kidney injury in rats mediating modulation of MAPK pathway," Molecular and Cellular Biochemistry, vol. 452, no. 1-2, pp. 141-152, 2019.

[62] S. Faubel, E. C. Lewis, L. Reznikov et al., "Cisplatin-induced acute renal failure is associated with an increase in the cytokines interleukin (IL)- $1 \beta$, IL-18, IL-6, and neutrophil infiltration in the kidney," Journal of Pharmacology and Experimental Therapeutics, vol. 322, no. 1, pp. 8-15, 2007.

[63] J. L. M. Digby, T. Vanichapol, A. Przepiorski, A. J. Davidson, and V. Sander, "Evaluation of cisplatin-induced injury in human kidney organoids," American Journal of PhysiologyRenal Physiology, vol. 318, no. 4, pp. F971-f978, 2020.

[64] N. Dixit and S. I. Simon, "Chemokines, selectins and intracellular calcium flux: temporal and spatial cues for leukocyte arrest," Frontiers in Immunology, vol. 3, p. 188, 2012.

[65] S. C. Zmonarski, M. Banasik, K. Madziarska, O. Mazanowska, and M. Krajewska, "The role of toll-like receptors in multifactorial mechanisms of early and late renal allotransplant injury, with a focus on the TLR4 receptor and mononuclear cells," Official Organ Wroclaw Medical University, vol. 28, no. 7, pp. 981-987, 2019.

[66] J.-J. Gao, G.-Y. Cai, Y.-C. Ning et al., "DAP5 ameliorates cisplatin-induced apoptosis of renal tubular cells," American Journal of Nephrology, vol. 35, no. 5, pp. 456-465, 2012.

[67] W. Cui, G. Hu, J. Peng, L. Mu, J. Liu, and L. Qiao, "Quercetin exerted protective effects in a rat model of sepsis via inhibition of reactive oxygen species (ROS) and downregulation of high mobility group box 1 (HMGB1) protein expression," Medical Science Monitor, vol. 25, pp. 5795-5800, 2019.

[68] S.-H. Kim, E.-S. Yoo, J.-S. Woo et al., "Antitumor and apoptotic effects of quercetin on human melanoma cells involving JNK/P38 MAPK signaling activation," European Journal of Pharmacology, vol. 860, Article ID 172568, 2019.

[69] C.-Y. Lan, S.-Y. Chen, C.-W. Kuo, C.-C. Lu, and G.-C. Yen, "Quercetin facilitates cell death and chemosensitivity through $\mathrm{RAGE} / \mathrm{PI} 3 \mathrm{~K} / \mathrm{AKT} / \mathrm{mTOR}$ axis in human pancreatic cancer 
cells," Journal of Food and Drug Analysis, vol. 27, no. 4, pp. 887-896, 2019.

[70] R. Zhang, X. Ai, Y. Duan et al., "Kaempferol ameliorates H9N2 swine influenza virus-induced acute lung injury by inactivation of TLR4/MyD88-mediated NF- $\kappa \mathrm{B}$ and MAPK signaling pathways," Biomedicine \& Pharmacotherapy, vol. 89, pp. 660-672, 2017.

[71] G.-J. Huang, S.-S. Huang, and J.-S. Deng, “Anti-inflammatory activities of inotilone from phellinus linteus through the inhibition of MMP-9, NF- $\kappa$ B, and MAPK activation in vitro and in vivo," PLoS One, vol. 7, no. 5, Article ID e35922, 2012.

[72] S. J. Holditch, C. N. Brown, A. M. Lombardi, K. N. Nguyen, and C. L. Edelstein, "Recent advances in models, mechanisms, biomarkers, and interventions in cisplatin-induced acute kidney injury," International Journal of Molecular Sciences, vol. 20, no. 12, 2019. 